\title{
Platelet Aggregation Amplitude Measurement
}

National Cancer Institute

\section{Source}

National Cancer Institute. Platelet Aggregation Amplitude Measurement. NCI Thesaurus. Code C111293.

The determination of the amount or magnitude of the platelet aggregation present in a sample. 